ISSN: 2386-3919 - e-ISSN: 2386-3927

DOI: https://doi.org/10.14201/et2016342109130

\title{
FORMACIÓN EN COMPETENCIAS COMUNICATIVAS DE LOS MAESTROS DE EDUCACIÓN INFANTIL
}

\author{
Training in communicative competences \\ of primary school teachers
}

José CARDONA ANDÚJAR

Departamento de Didáctica, Organización Escolar y Didácticas Especiales. UNED, Facultad de Educación

Correo-e: jcardona@edu.uned.es

Recibido: 3/11/2015; Aceptado: 12/6/2016; Publicado: 30/11/2016

Ref. Bibl. JOSÉ CARDONA ANDÚJAR. Formación en competencias comunicativas de los maestros de Educación Infantil. Enseñanza \& Teaching, 34, 2-2016, 109-130.

RESUMEN: En este artículo se presenta un trabajo de investigación descriptiva, desde la modalidad denominada encuesta transversal, utilizando un instrumento de corte cuantitativo, como es el cuestionario (válido y fiable), que permite un acceso rápido a los sujetos de la muestra. Ésta la componen un conjunto de maestros (75) de Educación Infantil que ejercen la profesión en centros educativos situados en la ciudad de Talavera de la Reina (Toledo) y su comarca. El objetivo de la investigación es conocer las percepciones que tienen los maestros de Educación Infantil del dominio de sus competencias disciplinares (comunicativas), y con base en este conocimiento fundamentar y describir líneas de mejora en su formación inicial (Grado) y permanente (durante el ejercicio de su profesión en los centros educativos).

Los resultados obtenidos evidencian un dominio aceptable de la competencia comunicativa. Si bien esto es así, se han registrado valoraciones medias iguales o inferiores a 3 (sobre un máximo de 4), con lo que podemos concluir que dicha formación presenta aspectos necesitados de mejora, en especial en lo que se refiere a las competencias comunicativas siguientes: a) Primera: Conocer el desarrollo del lenguaje y diseñar estrategias didácticas orientadas al enriquecimiento de las competencias comunicativas (21\%), y en la que se registra un $5 \%$ de respuestas con puntuación 2 (incompetencia), y b) Segunda: Conocimiento de los fundamentos 
JOSÉ CARDONA ANDÚJAR

FORMACIÓN EN COMPETENCIAS COMUNICATIVAS DE LOS MAESTROS DE EDUCACIÓN INFANTIL

lingüísticos y didácticos del aprendizaje de lenguas y evaluar su desarrollo y competencia comunicativa (49\%), con un $21 \%$ que se perciben incompetentes.

Palabras clave: competencias comunicativas; competencias disciplinares; educación infantil; encuesta transversal; investigación descriptiva.

SUMMARY: This article presents a descriptive research work from the modality called cross-sectional survey, using a quantitative instrument as is the (valid and reliable) questionnaire, which allows quick access to the subjects of the study sample. Sample is composed of a set of Primary School teachers $(n=75)$ who practice in schools located in Talavera de la Reina (Toledo, Spain) and its region. The aim of the research is to understand the perceptions of Primary School teachers about their proficiency in their disciplinary competences (communicative), and based on this knowledge, inform and describe areas of improvement in their initial (Grade) and continuing (during the exercise of their profession in schools) education.

The results show an acceptable command of communicative competence. While this is so, there have been equal or less than 3 (out of a maximum of 4) average ratings, so we can conclude that this training has some areas for improvement, particularly with regards to the following communicative competences: a) First: Understanding the language development and designing teaching strategies tailored to the enrichment of communicative competences (21\%), in which $5 \%$ of responses were recorded with a score of 2 (incompetence), b) Second: Understandingthe linguistic and educational foundations of language learning and assessing their development and communicative competence (49\%), with a $21 \%$ of responses perceived as incompetent.

Key words: communicative competences; disciplinary competences; primary education; cross-sectional survey; descriptive research.

\section{INTRODUCCIÓN}

Actualmente nos hallamos inmersos en proceso de reforma, que supone una nueva concepción de los contenidos profesionales orientados hacia un enfoque competencial (Sevillano, 2009; Cardona, 2013; Medina, 2014) a la hora de diseñar y desarrollar el currículo de las titulaciones en los distintos niveles del sistema educativo, incluido el universitario. En este marco aparece un reto fundamental: construir y aplicar un modelo de docencia más centrado en el desarrollo de competencias. En esta línea, se ha afirmado que las formas tradicionales de enseñar ya no sirven, ya que tanto la sociedad como el alumnado han cambiado (Cardona, 2008; Marcelo y Vaillant, 2009). A veces, se tiene la impresión de que la enseñanza y el aprendizaje escolar no se han transformado al mismo nivel que lo ha hecho una sociedad que, hoy día, se justifica y explica en el nuevo marco de los medios de comunicación y las nuevas tecnologías.

En armónica relación con el contenido del párrafo anterior, la última Ley Orgánica sobre Educación es meridianamente clara cuando, en su preámbulo, 
afirma: «Las habilidades cognitivas, siendo imprescindibles, no son suficientes; es necesario adquirir desde edades tempranas competencias transversales, como el pensamiento crítico, la gestión de la diversidad, la creatividad o la capacidad de comunicar, y actitudes clave como la confianza individual, el entusiasmo, la constancia y la aceptación del cambio", y subrayando a continuación que "necesitamos propiciar las condiciones que permitan el oportuno cambio metodológico, de forma que el alumnado sea un elemento activo en el proceso de aprendizaje. Los alumnos y alumnas actuales han cambiado radicalmente en relación con los de hace una generación» ( $B O E$ 295, de 10-XII-2013: LOMCE, preámbulo IV). Todo ello es aplicable, sin duda, al estudiante para futuro profesor.

En este sentido, parece necesario abordar acciones de investigación que nos trasladen hasta un perfil profesional del docente, en nuestro caso del maestro y la maestra de Educación Infantil, lo más acorde con la realidad de sus funciones y compromisos éticos, sociales y técnicos en una sociedad caracterizada por el cambio acelerado. Desde este estudio se trata de conocer la percepción que estos docentes tienen sobre su quehacer profesional en relación con algunas de las competencias específicas de su titulación, y que, entre otras, constituyen el bagaje necesario de formación para el desempeño de la labor docente en esta nuclear etapa educativa.

Presentamos este estudio estructurado en los siguientes grandes bloques: a) Una síntesis de la teoría que permita dar sentido e interpretar adecuadamente los datos que se obtengan durante el proceso de la investigación, b) Los aspectos metodológicos del trabajo (su naturaleza, población, muestra, etc.), c) El análisis de la percepción que los docentes manifiestan sobre su dominio de algunas competencias disciplinares (5) contempladas para el ejercicio docente en la etapa de Educación Infantil y d) Las conclusiones más relevantes que de nuestro estudio válidamente se deducen, proponiendo futuras, y necesarias, líneas de investigación al respecto.

\section{EL APRENDIZAJE POR COMPETENCIAS}

La formación basada en competencias parte de una serie de supuestas evidencias sobre las relaciones entre las instituciones educativas y el mundo del trabajo (Tejada y Navío, 2005). Se denuncia que la formación inicial para el trabajo sea insuficiente a la hora de satisfacer las demandas de los empleadores, criticándose la desconexión, a veces profunda, registrada entre la formación inicial recibida y el desempeño profesional (Zabalza, 2003). Sólo a través de esta formación, como han constatado diferentes investigaciones (Villa y Poblete, 2007; Sotomayor y Walker, 2009), no se garantiza la adquisición de las competencias básicas para el desempeño profesional docente, lo que deberá tenerse en cuenta tanto al diseñar la formación de Grado, como en aquellos programas de desarrollo profesional en ejercicio.

Aunque asumamos que la formación inicial del maestro (Grado) es sólo una de las etapas de su desarrollo profesional, la incidencia de dicha formación de 
entrada estará en función de cómo se conciba la tarea de esos profesionales sobre un alumnado a quien la sociedad demanda una capacitación acorde a los cambios que en ella se producen. Con base en esta transformación innegable de la sociedad actual, y la del futuro inmediato, deben emerger y consolidarse modelos innovadores de formación del profesorado en general, y de los maestros de infantil en particular. En este sentido, hace ya tiempo que Hargreaves (2005) advertía que los docentes perciben que su profesión está cambiando, modificación provocada por la mutación de la sociedad en la que lo desempeñan.

Bunk aporta nuevos criterios de demarcación y validación del trabajo profesional, al afirmar que "posee competencia profesional quien dispone de conocimientos, destrezas y aptitudes necesarias para ejercer una profesión, puede resolver los problemas profesionales de forma autónoma y flexible, y está capacitado para colaborar en su entorno profesional y en la organización del trabajo» (1994: 9). En esta dirección han insistido diversos autores (Levi-Leboyer, 1998; Tejada, 1999a; Beduwé y Planas, 2003; Castillo, Rubio y Cabrerizo, 2007; Sevillano, 2009; Blanco, 2010; Medina, 2014).

Volviendo a Bunk (1994), una adecuada articulación de saber incluirá: a) Una competencia técnica (saber), en la que se domine como experto las tareas y contenidos de su ámbito laboral, y los conocimientos y destrezas necesarios para ello; b) Una competencia metodológica (saber hacer), que supone un saber reaccionar, aplicando los procedimientos adecuados, a las tareas encomendadas y a las irregularidades que se presenten, encontrando de forma independiente vías de solución y transfiriendo adecuadamente las experiencias adquiridas a otros problemas de trabajo; c) Una competencia social (saber ser), que permita colaborar con otras personas de forma comunicativa y constructiva, mostrando un comportamiento orientado al grupo, favoreciendo de esta manera el entendimiento interpersonal, y d) Una competencia participativa (saber estar), que conlleva participar en la organización de su puesto de trabajo y su entorno laboral, capacitando para labores organizativas y toma de decisiones, estando dispuesto a aceptar responsabilidades.

\section{UNA APROXIMACIÓN AL CONCEPTO DE COMPETENCIA}

Frente a quienes afirman la falta de una definición coherente del concepto (Mulder et al., 2008), la competencia (Tejada, 1999b; Coll, 2007) integra el saber, saber hacer, saber estar y saber ser, y se define, en una primera aproximación al concepto, como un conjunto de conocimientos, procedimientos y actitudes que han de estar combinados, coordinados e integrados en el ejercicio profesional.

La competencia podemos entenderla (De Miguel, 2005) como el conjunto de capacidades y de saberes técnicos, metodológicos, sociales y participativos que la persona pone en juego para afrontar las exigencias que le plantea el mundo laboral. Asimismo, ha gozado de consenso la clásica definición que 
aportó en su día la OIT (1998), al entenderla como aquella capacidad de integrar lo intelectual y lo emocional en términos de conocimientos, habilidades, actitudes y prácticas.

A pesar de lo anterior, vivimos una época proclive a la mitificación del término competencia, siendo numerosas las aportaciones que buscan dotar de significación a este significante de novedosa utilización (Tejada, 1999a). Al parecer (Rodríguez, 2006, citado en Sevillano, 2009: 5), la intersección conceptual contiene un conjunto de elementos comunes a todas ellas, ya que: a) Son características permanentes de la persona; b) Se ponen de manifiesto cuando se ejecuta una tarea; c) Están relacionadas con la ejecución exitosa de una actividad, ya sea laboral o de otra índole; d) Tienen una relación causal con el rendimiento laboral, es decir, no están sólo asociadas con el éxito, sino asumiendo que realmente lo causan, y e) Pueden ser generalizables a más de una actividad profesional.

En el mundo del trabajo, se ha definido como la capacidad de la persona de integrar lo intelectual y emocional con base en conocimientos, habilidades y prácticas necesarias para desempeñar una función de manera eficaz, eficiente y creativa en armonía con la demanda de una tarea concreta. Esta finalidad la encontramos, asimismo, respecto a la incidencia en el ámbito de la formación y, más específicamente, en el de la educación en las instituciones docentes (Delors, 1996). En tal sentido, Delors defiende que los procesos educativos deben facilitar en los educandos la adquisición de competencias que les permitan aprender a hacer, a conocer, a ser y a vivir juntos.

No obstante, sea cual fuere el ámbito en el que nos situemos (laboral, educativo o artístico), en el concepto de competencia convergen una serie de ideas que contribuyen a configurar el concepto que nos ocupa, tales como a) Conocimientos, actitudes y valores; b) Movilizar capacidades diversas, y c) Desempeño en contextos plurales a través de una actuación adhocrática (Irigoin y Vargas, 2002). En el segundo de ellos (educativo), y a tenor del nuevo marco que preside los procesos de enseñanza y aprendizaje, desde la llamada segunda revolución industrial (electrónica, tecnología computacional) emergen demandas urgentes que (Sevillano, 2009) se orientan a tener que repensar todo, a dotar de importancia nuclear a la formación permanente y hasta asumir cambios en (repensar) la misión de la escuela.

La competencia de los individuos se deriva, pues, de la posesión de una serie de atributos (conocimientos, valores, habilidades y actitudes) que se utilizan de forma combinada para el desempeño del trabajo (Gonczi, 1996; Montero, 2010). Con este referente adquieren sentido algunas definiciones de competencia profesional, entre las que destaca, como la más generalizada y aceptada, la siguiente: saber hacer en un contexto. Por saber hacer se hace referencia: a) A repertorios de comportamientos que algunas personas dominan y que las hace eficaces en una situación determinada o b) A la posesión de conocimientos, habilidades y actitudes para realizar una tarea o desempeñar un papel de manera adecuada para lograr un resultado deseado (Angulo, 2003). 
Entre las múltiples aportaciones que han ido configurando el concepto de competencia, se pueden encontrar definiciones en el Proyecto Tuning Educational Structures in Europe ${ }^{1}$ (González y Wagennar, 2003), en el informe DeSeCo (Definition and Selection of Competencies: Analysis of Theoretical and Conceptual Foundations) realizado por la OCDE, en el Informe Delors (1996), así como en la propuesta para la elaboración de Títulos Universitarios de Grado y Máster (MEC, 2006), o en diferentes autores que han realizado importantes contribuciones en los últimos años (Zabalza, 2005; Cabero, 2005; Barnett, 2005; Rué y Martínez, 2005; Roegiers, 2007). Según Perrenoud (2000 y 2007), la competencia es la capacidad de la persona para movilizar diversos recursos cognitivos que le permitan enfrentar un tipo de situaciones.

La definición de competencia adoptada por Roegiers (2007) subraya el aspecto de recursos que se movilizan ante una situación e introduce la idea de familias de situaciones para trabajar las competencias en la escuela. Zabala y Arnau (2008) analizan distintas definiciones de competencia, de autores tanto del mundo laboral como del entorno educativo. Con base en este análisis definen competencia como "la capacidad o habilidad de efectuar tareas o hacer frente a situaciones diversas de forma eficaz en un contexto determinado, y para ello es necesario movilizar actitudes, habilidades y conocimientos al mismo tiempo y de forma interrelacionada" (Zabala y Arnau, 2008: 43-44).

Existe, como se ve y decíamos ya, una zona de intersección entre los conjuntos que conforman las diferentes visiones sobre el significado de la competencia como concepto, lo que facilita conocer aquellos parámetros que esencialmente la caracterizan. Hay, pues, consenso al afirmar que "las competencias docentes son el conjunto de conocimientos, habilidades y actitudes, en calidad de recursos necesarios para resolver adecuadamente las situaciones a las que se enfrentan, en su quehacer profesional, los profesores, y su adquisición precisa una interrelación armónica entre formación teórica y la aplicación de la misma en la realidad de las instituciones educativas» (Cardona, 2013: 91).

\section{FORMACIÓN EN COMPETENCIAS EN EDUCACIÓN INFANTIL}

La labor del maestro se debe desarrollar en un clima de colaboración, practicando una dinámica de coordinación entre colegas que favorezca el intercambio de experiencias, innovaciones y dificultades, al tiempo que permite asegurar a su

1. Proyecto que, como se sabe, uno de sus elementos más característicos es la aplicación de una particular metodología de trabajo, que cuenta con cuatro grandes ejes de actuación y con la colaboración de varios equipos de expertos: 1) Competencias genéricas, 2) Competencias disciplinarias específicas, 3) El papel del sistema ECTS como sistema de acumulación y 4) La función del aprendizaje, la docencia, la evaluación y el rendimiento en relación con el aseguramiento y la evaluación de la calidad (http://eees.universia.es/europa/). 
alumnado una coherencia o, al menos, cierta continuidad en su proceso formativo. Sin embargo, se pretenden potenciar todas estas habilidades profesionales de carácter cooperativo a través de una cultura universitaria academicista e individualista (Zabala, 2007). Frente a ello, las nuevas necesidades de aprendizaje discente exigen unas innovadoras maneras de enseñar (Tobón, 2005; Cardona, 2013) y esto conlleva innovaciones a la hora de formar a los profesores.

En la actualidad, a los docentes se les ha elevado su estatus profesional y ya no aparecen como tecnólogos educativos que se limitan a ejecutar las disposiciones que dictaminan las distintas administraciones educativas. Por el contrario, se apuesta por un profesional autónomo, práctico-reflexivo, investigador, con capacidad para tomar decisiones sobre los aspectos más relevantes de su propia práctica. Se aboga, en consecuencia, por un profesional autocrítico, capaz de valorar en colaboración sus actuaciones y de mejorar todas aquellas dimensiones profesionales menos ajustadas a las finalidades previstas. Se está hablando, por tanto, de nuevas competencias para maestros y profesores. En este enfoque, tanto la formación inicial como la continua de los maestros de Educación Infantil en aquellas competencias que aparecen como imprescindibles para su práctica profesional está fuera de toda discusión. Es, como afirman García y Murga, «una tarea ineludible» (2015: 126).

\subsection{Síntesis del catálogo de competencias en Educación Infantil}

ANECA elaboró una fundamentada taxonomía de competencias profesionales del perfil de maestro, aportación que constituye un referente esencial para el presente trabajo de investigación. Dicha propuesta, incluida en el Libro Blanco: Título de Grado en Magisterio. Volumen 1 (ANECA, 2004), surge de la propia epistemología de la teoría de competencias del proyecto Tuning y de la especificidad de las competencias en relación a la profesión docente. Son tres tipos de competencias los que el estudio de ANECA presenta. A saber: a) Transversales o genéricas, b) Específicas docentes y c) Especializadas.

El citado Libro Blanco precisa y relaciona las competencias específicas en Educación Infantil pensando en un docente de perfil generalista, fundamentado en las características educativas derivadas del principio de globalización de la actividad docente propias de esta etapa educativa. En la clasificación se destaca la importancia del lenguaje, el desarrollo de habilidades, técnicas y procedimientos para saber hacer las cosas; así como la globalización de elementos, la observación y la comunicación desde la perspectiva psicológica, todo ello para el alumnado de Educación Infantil y el profesorado que lo atiende.

Según el ya referido Libro Blanco, de la relación de treinta competencias docentes específicas comunes contempladas para el profesional de Educación Infantil y que integran los conocimientos disciplinares (saber), profesionales (saber hacer) y académicos, aquellas que obtuvieron la máxima valoración fueron las cinco que se incluyen en la Tabla 1 que se adjunta. 
JOSÉ CARDONA ANDÚJAR

FORMACIÓN EN COMPETENCIAS COMUNICATIVAS DE LOS MAESTROS DE EDUCACIÓN INFANTIL

TABLA 1

Competencias docentes más valoradas

\begin{tabular}{|l|c|}
\hline \multicolumn{1}{|c|}{ COMPETENCIAS ESPECÍFICAS DE EDUCACIÓN INFANTIL } & VALOR \\
\hline $\begin{array}{l}\text { Conocer el desarrollo del lenguaje en la etapa de Educación } \\
\text { Infantil y diseñar estrategias didácticas orientadas al } \\
\text { enriquecimiento de las competencias comunicativas }\end{array}$ & 3.58 \\
\hline $\begin{array}{l}\text { Conocer y promover el desarrollo cognitivo, social y de la personalidad } \\
\text { desde el nacimiento hasta los primeros años de la escolarización obligatoria }\end{array}$ & 3.56 \\
\hline $\begin{array}{l}\text { Ser capaz de utilizar la observación sistemática como principal instrumento de } \\
\text { evaluación global, formativa y continua de las capacidades de los alumnos }\end{array}$ & 3.36 \\
\hline $\begin{array}{l}\text { Guiarse por el uprincipio de la globalización» a la hora de } \\
\text { programar actividades y tareas educativas de 0 a 6 años }\end{array}$ & 3.33 \\
\hline $\begin{array}{l}\text { Saber utilizar el juego como principal recurso didáctico, así como } \\
\text { diseñar actividades de aprendizaje basadas en principios lúdicos }\end{array}$ & 3.27 \\
\hline
\end{tabular}

Fuente: ANECA, 2004: 95.

Desde las anteriores coordenadas, y habida cuenta de las características y consideraciones en la capacitación de los nuevos docentes a la luz del EEEs, cabría resaltar lo que esencialmente se exige del maestro/a con perfil en Educación Infantil en el marco del título de grado pertinente. Centrándonos en las específicas, contempla lo que han de saber estos profesionales (conocimiento disciplinar), lo que han de saber realizar (competencias profesionales) y lo que han de dominar (competencias académicas).

Este informe ANECA ha servido de base para la elaboración del cuestionario (Anexo I) utilizado en la presente investigación. En él se han incorporado cinco específicas comunes, cinco disciplinares (competencias) y cinco profesionales, si bien sólo las relativas al conocimiento disciplinar (capacidad comunicativa) son incluidas y analizadas en el presente trabajo. En este sentido, han sido elegidas aquellas cinco competencias pertenecientes al área de lingüística que fueron más valoradas en dicho informe y que se relacionan en la Tabla 2 (incluyéndose el valor asignado), e incorporándose al cuestionario. Ellas fundamentan el desarrollo del presente trabajo.

\section{Metodología}

Mejorar la educación exige, entre otras actuaciones, repensar la formación del profesorado buscando nuevos enfoques en la fase inicial de la misma. Para intentar responder algunos de los interrogantes que ello plantea se ha seguido el siguiente diseño metodológico. 
JOSÉ CARDONA ANDÚJAR

FORMACIÓN EN COMPETENCIAS COMUNICATIVAS DE LOS MAESTROS DE EDUCACIÓN INFANTIL

TABLA 2

Competencias disciplinares en lingüística

\begin{tabular}{|l|c|}
\hline \multicolumn{1}{|c|}{ COMPETENCIA } & VALOR \\
\hline $\begin{array}{l}\text { 1. Conocer el desarrollo del lenguaje y diseñar estrategias didácticas } \\
\text { orientadas al enriquecimiento de las competencias comunicativas. }\end{array}$ & 3.58 \\
\hline $\begin{array}{l}\text { 2. Conocer los fundamentos lingüísticos y didácticos del aprendizaje de } \\
\text { lenguas y evaluar su desarrollo y competencia comunicativa. }\end{array}$ & 2.99 \\
\hline $\begin{array}{l}\text { 3. Conocer la literatura infantil y desarrollar estrategias para el } \\
\text { acercamiento de los niños al texto oral como escrito. }\end{array}$ & 2.86 \\
\hline $\begin{array}{l}\text { 4. Dominar las lenguas oficiales de su comunidad y mostrar una correcta } \\
\text { producción y comprensión lingüística. }\end{array}$ & 3.10 \\
\hline $\begin{array}{l}\text { 5. Promover la incorporación de los niños al aprendizaje funcional de una } \\
\text { lengua extranjera. }\end{array}$ & 2.18 \\
\hline
\end{tabular}

Fuente: ANECA, 2004.

\subsection{El problema}

El trabajo de investigación que aquí se presenta persigue aportar información que contribuya al futuro diseño de un nuevo modelo formativo que se oriente no sólo a la adquisición de conocimientos por parte del estudiante para profesor, sino al desarrollo de una serie de competencias entendidas, se ha dicho con anterioridad, como capacidades, destrezas, conocimientos... en función de los correspondientes perfiles académicos y profesionales. El objetivo de la formación de docentes es algo más complejo que el dominio o la transmisión de conocimientos, como así ha sucedido tradicionalmente, ya que la educación pasa de estar centrada en la enseñanza (dimensión instrumental o magistrocéntrica), a estarlo en el aprendizaje (dimensión esencial o sustantiva del acto educativo). En dichas coordenadas, conocer cuál es el nivel competencial actual del docente de Educación Infantil (exclusivamente en lo disciplinar para este informe) es el problema, o interrogante, del que partimos aquí.

\subsection{La finalidad}

En el sentido apuntado en el epígrafe anterior, nuestra intención es indagar sobre dicho perfil competencial del profesorado de Educación Infantil, ya que lo consideramos muy relacionado con un desempeño eficaz de la función docente en la realidad escolar actual, lo que ha de informar el diseño de la formación universitaria de estos profesionales al objeto de intervenir en la misma y mejorarla. Por todo ello, nos hemos centrado en el estudio en un campo sobre el que nos parece necesario desarrollar acciones de investigación que nos aproximen hasta el citado modelo formativo del Maestro de Educación Infantil, modelo que sea lo más acorde posible con sus funciones y compromisos tanto éticos, como sociales y técnicos, en el marco de un aprendizaje basado en competencias. 
Es, pues, un estudio de exploración y descubrimiento, en el que la problemática que lo justifica y fundamenta se establece en indagar-constatar la presencia, y a qué nivel, de los conocimientos, aptitudes y destrezas (competencias) que han debido adquirirse por un grupo de profesionales para permitir la optimización del proceso de enseñanza y aprendizaje en Educación Infantil.

\subsection{Tipo de investigación}

La nuestra es una investigación desde un enfoque descriptivo-interpretativo mediante el empleo de instrumentos de corte cuantitativo, como es el cuestionario, que permite un tratamiento de la realidad mediante el contraste de las percepciones que tienen los maestros/as de Educación Infantil con respecto a algunas de sus competencias docentes. Para ello nos basamos en la encuesta y, más concretamente, en la encuesta transversal, pues nos va a permitir acceder rápidamente a una muestra de sujetos de una sola vez y durante un corto periodo de tiempo, con el objetivo de captar ciertos fenómenos presentes en el momento concreto de su realización (Anguera, 1995).

La metodología por encuesta, fundamentada en una muestra significativa de la población o universo determinado, presenta una serie de ventajas que debidamente encajan-armonizan con los objetivos que intentamos alcanzar, y que son (Cardona, 2008): a) Los resultados que se obtienen desde ella pueden ser generalizados a una población definida por ser mayor el número de elementos que conforman la muestra que en otras metodologías; b) Es apropiada para recoger opiniones, creencias o actitudes, y c) Supone una alternativa válida cuando el investigador no puede acceder a toda la población.

Los estudios de encuesta tratan de describir la naturaleza de las condiciones existentes en un colectivo, identificar valores estándar con los que poder comparar y determinar las relaciones existentes entre eventos específicos (Colás y Buendía, 1994). Otra de las ventajas que presentan los estudios de encuesta, mediante el uso y aplicación de cuestionarios, es que no solamente se puede llegar a caracterizar a una población determinada, sino que también permite llegar a establecer comparaciones entre los sujetos estudiados, si bien en función de las variables (estratos) establecidas en el pertinente cuestionario (Bisquerra, 2004).

Además, nuestro trabajo es una investigación ex post facto, ya que cumple aquellas características más esenciales que definen un estudio de la naturaleza del nuestro: no se puede manipular la variable independiente (formación competencial) ni se han seleccionado los sujetos de la muestra en función del dominio o no de la misma, sino que ésta es ya una realidad dada.

\subsection{Población y muestra}

En nuestra investigación, de una población de 80 sujetos (docentes de infantil que ejercen en los CEIP de la zona de Talavera de la Reina, Toledo), la muestra ha 
sido seleccionada aplicando un muestreo aleatorio estratificado (maestra y maestro) y mediante la pertinente afijación proporcional, esto es, que el número de elementos muestrales de cada estrato sea directamente proporcional al tamaño del estrato dentro de la población.

\section{CUADRO I}

Cálculo para hallar la muestra representativa de la población estudiada

$$
\mathrm{n}=\mathrm{Z}^{2} \mathrm{z} \cdot \mathrm{p} \cdot \mathrm{q} \cdot \mathrm{N} / \mathrm{e}^{2}(\mathrm{~N}-1)+\mathrm{Z} \mathrm{z}^{2} \cdot \mathrm{p} \cdot \mathrm{q}=2^{2} \cdot 83 \cdot 17 \cdot 80 / 9(80-1)+
$$

Donde: $\mathrm{n}=$ Número de elementos que debe poseer la muestra $/ \mathrm{z}=$ Nivel de Significación / $\mathrm{Zz}=$ Puntuación correspondiente al nivel de significación $/ p=\%$ estimado $\left(^{*}\right) / q=100-p / e$ $=$ error permitido

$\left({ }^{*}\right) \mathrm{Al}$ no conocer previamente el porcentaje de presencia en la población de las características estudiadas, se procedió a un estudio inicial sobre una muestra pequeña (14 sujetos). El resultado de la misma, en base a la frecuencia de puntuaciones superiores a la media de la banda de valoración (2,5 puntos), fija esta presencia ( $p$ ) en el $83 \%$, correspondiendo para "q" un índice del $17 \%$.

De esta forma, y tras la selección aleatoria, se obtuvo una muestra invitada compuesta por 75 docentes, superando el número mínimo de 71 sujetos considerados necesarios para ser ésta estadísticamente representativa para un margen de

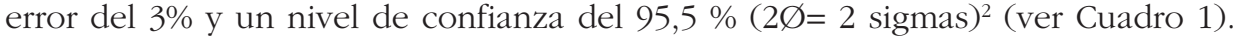
Subrayar que la formación inicial de este colectivo (Diplomatura, en la mayoría de los casos) no fue diseñada propiamente para el desarrollo de competencias, tal y como ocurre en la actualidad con la titulación de Grado en Educación Infantil. En la siguiente Tabla 3 podemos ver la población y muestra por estratos.

TABLA 3

Muestra obtenida para la presente investigación

\begin{tabular}{|c|c|c|c|}
\hline INFANTIL $\left(2{ }^{\circ} \mathrm{C}\right)$ & MAESTRAS & MAESTROS & TOTAL \\
\hline \multirow{2}{*}{ Población } & 59 & 21 & 80 \\
& $(74 \%)$ & $(26 \%)$ & $(100 \%)$ \\
\hline \multirow{2}{*}{ Muestra } & 55 & 20 & 75 \\
& $(74 \%)$ & $(26 \%)$ & $(100 \%)$ \\
\hline
\end{tabular}

2. Entre +2 y -2 sigmas de la curva de distribución normal de Gauss, a partir de la media, está incluido el 95,5\% de la población. Esto quiere decir que tenemos una probabilidad de que 955/1000 coincidan con los de la población total. 


\subsection{Técnica e instrumento}

La recopilación de los datos, como queda dicho, se ha llevado a cabo mediante el cuestionario (ver anexo), herramienta adecuada para, como es el caso, conseguir información acerca de un fenómeno social (Martínez, 2002) como lo es la formación en la competencia comunicativa de los maestros de infantil. Hoy en día el cuestionario es la técnica de recogida de datos más utilizada en la investigación por encuesta. Es uno de los métodos más comunes para obtener datos estadísticos acerca de una gran variedad de temas (Bisquerra, 2004), con propósitos de investigación, y que se lleva a cabo en distintas disciplinas como, por ejemplo, la educación como apuntamos. A través del cuestionario se pretende conocer lo que hacen, opinan o piensan los encuestados mediante preguntas realizadas por escrito y que pueden ser respondidas sin la presencia del encuestador (Buendía, 1999).

Para su elaboración hemos seguido fielmente los pasos habituales en estos casos y que son (Martínez, 2002): reflexionar y definir la información necesaria, realizar una redacción adecuada (clara y precisa) de las preguntas (se ha respetado aquí la redacción ANECA), y el tipo de las mismas (cerradas en nuestro caso), introducción (aunque se suprime en anexo por razones de espacio) al mismo, las instrucciones pertinentes, y decidir el diseño de su aspecto formal.

En lo que se refiere a la fiabilidad del cuestionario utilizado (consistencia interna), ésta se ha establecido mediante el Alfa de Cronbach, toda vez que es una técnica que facilita dicho objetivo desde el requerimiento de una sola aplicación del instrumento, con base en la medición de la respuesta de los sujetos respecto a las preguntas (ítems) del instrumento aplicado; este estadístico informa de la estabilidad o consistencia de los resultados obtenidos. Con este fin se aplicó el cuestionario a diez sujetos de la población elegida, dando lugar a un conjunto de datos que, una vez analizados, nos proporcionaron los siguientes resultados (véase Cuadro 2).

CUADRO II

Coeficiente de fiabilidad del cuestionario

\section{ESTADÍSTICOS}

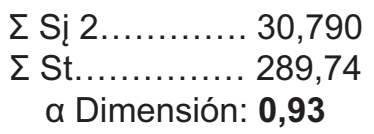

De los datos que se desprenden de los pertinentes procedimientos estadísticos utilizados para medir la fiabilidad (el Alfa de Cronbach), se deduce que los resultados de las pruebas aplicadas al cuestionario son buenos, por lo que se puede concluir con fundamento que el instrumento que se ha empleado para la recogida de información es válido (autoridad ANECA) y fiable. 


\subsection{Tratamiento y análisis de los datos}

Para el análisis de los datos, una vez que se recibieron los cuestionarios en papel, se procedió a su tratamiento y proceso mediante la introducción de los mismos en una base del programa Microsoft Excel (Paquete Microsoft Office XP). El análisis estadístico de los datos se ha realizado con el programa estadístico sPss versión 11.5 para Windows.

\section{PRESENTACIÓN Y ANÁLISIS DE DATOS}

Su presentación se lleva a cabo mediante los gráficos correspondientes, optando por uno de ellos para cada una de las variables aquí investigadas. Se ha considerado que este procedimiento proporciona la información deseada y permite la visión rápida (intuitiva) de los resultados obtenidos en las diferentes competencias.

\subsection{Competencias disciplinares (comunicativas)}

La primera de las competencias analizadas es «conocer el desarrollo del lenguaje y diseñar estrategias didácticas orientadas al enriquecimiento de las competencias comunicativas». La distribución de las frecuencias en el gráfico denota cómo la mayoría de los maestros/as se sitúa en las categorías numéricas 3 y 4, como un referente del perfil competencial de estos profesionales, tanto en evidencia intuitiva como en términos de análisis estadístico. En la distribución de porcentajes (Gráfico 1), puede apreciarse que la mayoría de respuestas se ubican en valores de bastante competente (valor 4), con un 74\%; seguido de los valores de competente (valor 3), con un $21 \%$, y poco competente (valor 2), con un 5\% de las respuestas.

GÁFICO 1

Competencias disciplinares

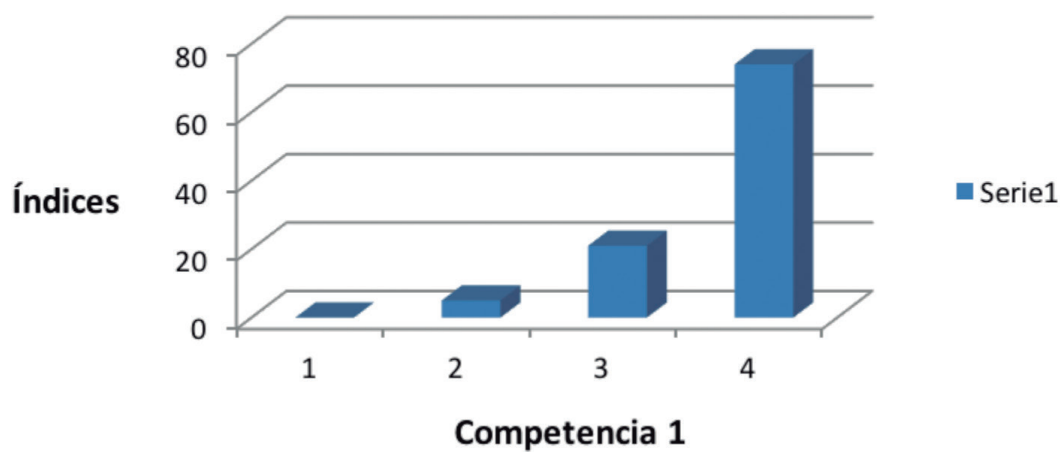


La segunda de las competencias estudiadas en la investigación es «conocer los fundamentos lingüísticos y didácticos del aprendizaje de lenguas y evaluar su desarrollo y competencia comunicativan; en ella la moda es 3, con un $49 \%$ del total de las respuestas (competentes); se ven bastante competentes (valor 4) el 30\% de los encuestados, mientras que los docentes que se consideran incompetentes son el 21\% de los casos (Gráfico 2).

GRÁFICO 2

Competencias disciplinares

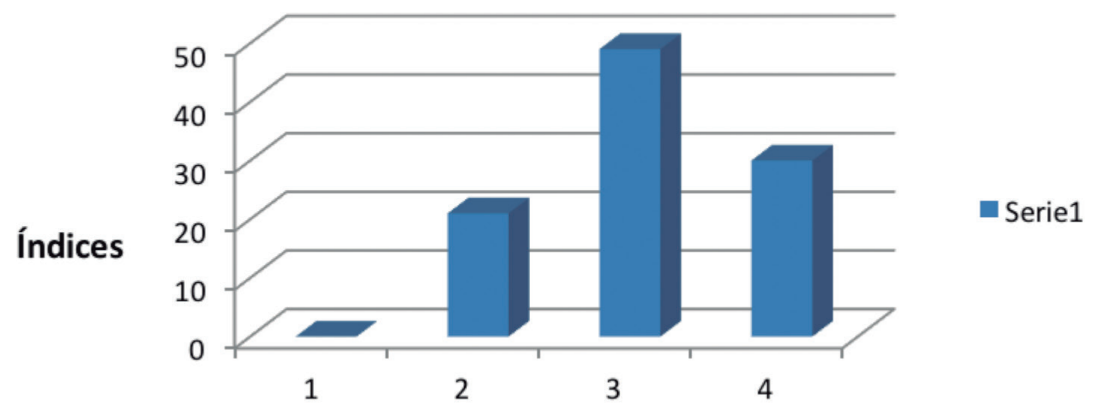

Competencia 2

Otra de las competencias exploradas (la tercera) relacionada, asimismo, con los conocimientos disciplinares es «conocer la literatura infantil y desarrollar estrategias para el acercamiento de los niños al texto tanto oral como escrito». La valoración o puntuación media que se registra fija la moda en el valor 3, lo que evidencia el desarrollo moderadamente mejorable de la misma por parte de los docentes de infantil. Las distribuciones de frecuencias asignan el $40 \%$ al valor 4 , el $60 \%$ al valor 3, no obteniéndose puntuación alguna en los valores dos y uno (Gráfico 3).

GRÁFICO 3

Competencias disciplinares

Índices

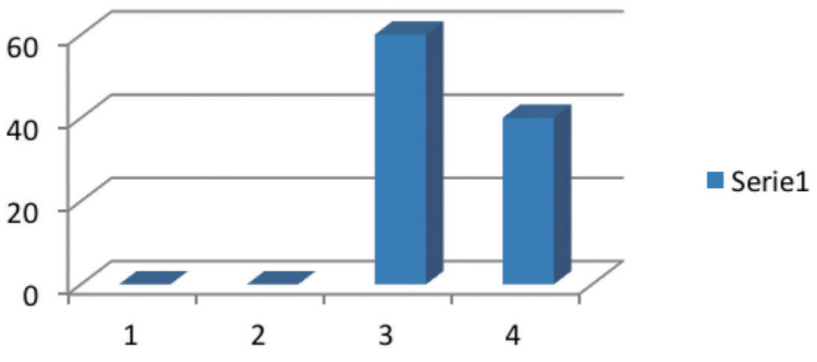

Competencia 3 
Tras analizar los datos relativos a la cuarta de las competencias objeto de estudio (esto es, la de «dominar las lenguas oficiales de su comunidad y mostrar una correcta producción y comprensión lingüística»), la moda se sitúa, asimismo, en el valor 3 (el 65\% de los encuestados ha marcado esta puntuación), lo que refleja que es también moderadamente mejorable la formación docente a la hora de favorecer entre el alumnado el dominio en expresión y comprensión de la lengua. A destacar el 33\% de maestros/as que se percibe como muy competente en ella (puntuación 4), registrándose sólo un 2\% de incompetencia.

GRÁFICO 4

Competencias disciplinares

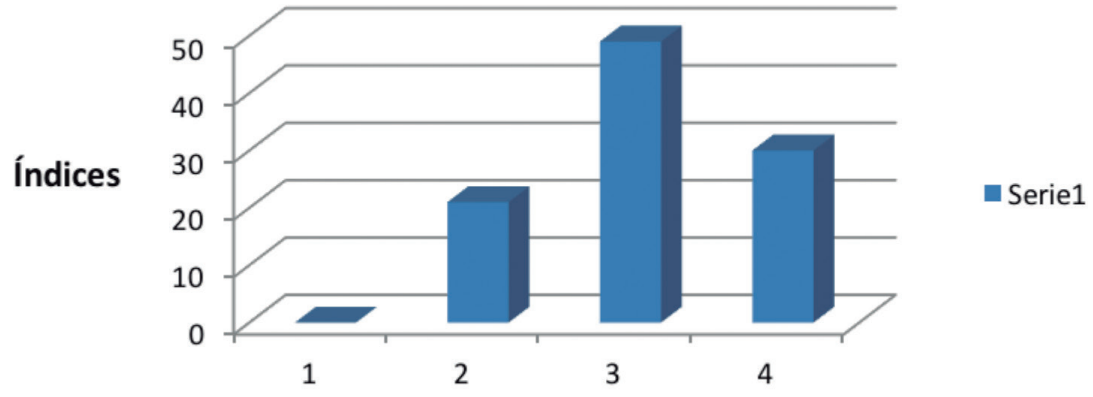

\section{Competencia 4}

La última competencia (la quinta) de las analizadas de este bloque es "promover la incorporación de los niños al aprendizaje funcional de una lengua extranjera». La media obtenida es de 3,78 y una moda de 4, con lo que la mayoría de los maestros/as se sitúan en la categoría 4 (es decir, bastante competentes). Esto indicaría que el profesorado esté cada vez más concienciado de la importancia de adquirir la formación en este ámbito disciplinar, lo que le permitirá mediar con solvencia en el aprendizaje de una lengua extranjera por parte del alumnado. En la distribución de frecuencias puede apreciarse como no hay valor 1 , y que el valor 2 sólo alcanza un 3\%. La mayoría de respuestas se ubican en valores de bastante competente (valor 4), con un 79,00\%, seguido de los valores de competente (valor 3) con un $18,00 \% \mathrm{y}$, por tanto, moderadamente mejorable su preparación en el dominio de esta competencia (Gráfico 5). 
JOSÉ CARDONA ANDÚJAR

FORMACIÓN EN COMPETENCIAS COMUNICATIVAS DE LOS MAESTROS DE EDUCACIÓN INFANTIL

GRÁFICO 5

Competencias disciplinares

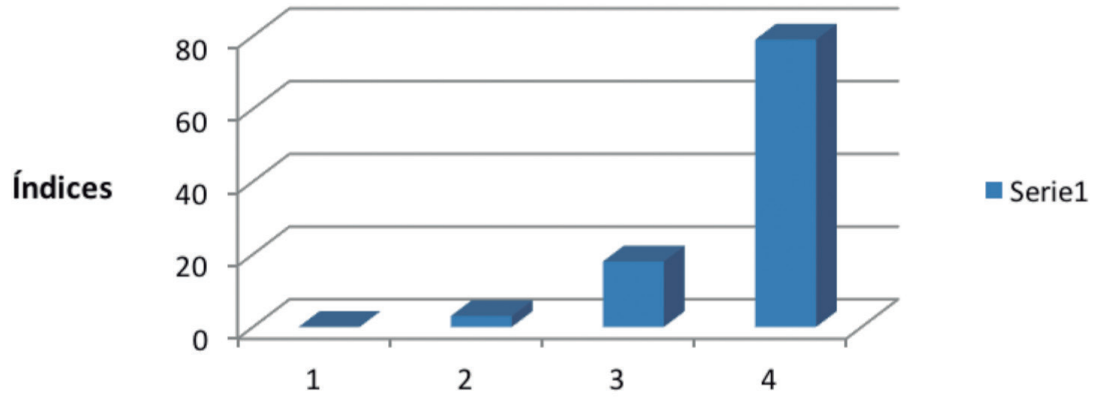

Competencia 5

\section{CONCLUSIONES Y PERSPECTIVAS DE FUTURO}

Con respecto a las competencias relacionadas con el ámbito disciplinar objeto de estudio del presente trabajo, significar que, siempre fundamentados en la propia (y exclusiva) percepción de los maestros y maestras, es, según los resultados, aceptable la formación de los docentes encuestados respecto del dominio de la competencia comunicativa, así como, específicamente, en lo referido a la aproximación mediadora en el aprendizaje de una lengua extranjera. Si bien esto es así, se han registrado valoraciones de su formación igual, o inferior, a 3 (sobre un máximo de 4), con lo que podemos concluir que dicha formación es, aunque sea moderadamente como ya decimos, susceptible de mejora.

Lo anterior no debe llevar a desmerecer a los docentes de la población investigada, ya que son numerosos los trabajos de investigación en este campo que concluyen con unas valoraciones similares a las que aquí se llega, e incluso con resultados, a veces, bastante más negativos, pues toda acción humana, incluyendo la de los profesionales de la docencia en general, y la de los maestros de infantil en particular, es siempre perfectible (véanse al respecto, y entre otras, las aportaciones de Vaillant, 2007; Álvarez y Fernández Cruz, 2009; o Marcelo, 2011).

De manera que una formación suficiente (con valor 3, indicador, como decimos, de un evidente, aunque moderado, margen de mejora) se ha registrado con frecuencia en nuestro trabajo de campo. Así: a) El 21\% de los docentes en la competencia primera, b) El 49\% en la segunda, c) El 60\% en la tercera competencia, d) El $65 \%$ en la cuarta, y e) Un 18\% en la quinta, última de las competencias analizadas. Pero, además, y si contemplamos (hay que hacerlo) las puntuaciones con un valor 2 , que denota incompetencia, tenemos registrado hasta un 5\% en la competencia primera (esto es, "conocer el desarrollo del lenguaje y diseñar estrategias didácticas orientadas al enriquecimiento de las competencias comunicativas") y el 21\% (uno de cada cinco) en la segunda ("conocer los fundamentos lingüísticos y didácticos 
del aprendizaje de lenguas y evaluar su desarrollo y competencia comunicativa"). Esto último invita a una revisión de los planes de formación para este colectivo.

Por tanto, y a la vista de los resultados obtenidos (aunque satisfactorios en general), avalados por otras investigaciones (Lomas et al., 1993; Pilleu, 2001; Bigas y Correig, 2008; Prado, 2011; Martín, 2013, entre otros), tratar de optimizar la formación de los docentes de la población estudiada es un desafío, un objetivo a tener en cuenta (respecto de las competencias comunicativas, en el nuestro). Para Martín (2013), la principal finalidad de la enseñanza de la lengua tiene en el aprendizaje de la correcta aplicación de la competencia comunicativa su principal objetivo, en tanto que constituye uno de los aspectos más relevantes de la lingüística aplicada.

Llegados hasta aquí, parece muy conveniente recordar que cuando hablamos de la competencia comunicativa nos referimos a un tipo de capacidad que comprende no sólo la habilidad lingüística de producir frases bien construidas, sino que han de considerarse también un conjunto de habilidades extralingüísticas de naturaleza social (Berruto, 1979), como puede ser el caso de las implicaciones de todo mensaje, o el sentido implícito o intencional del emisor en un contexto determinado, esto es, lo que el emisor quiere realmente decir con lo que dice. Y esto por citar sólo algunas de ellas.

Todo lo anterior cobra su auténtico sentido si se considera (y asume) que las competencias asociadas con el desarrollo y adquisición del lenguaje oral, y de la escritura, en el alumnado de Educación Infantil son fundamentales en la formación de los docentes, lo que les permitirá enfrentar la enseñanza de la lectoescritura, por poner solo un ejemplo, mediante el desarrollo de la competencia comunicativa, lo que favorece en el alumnado una serie de capacidades, entre las que se encuentra utilizar la lengua como instrumento útil de comunicación, de representación, aprendizaje y disfrute, de expresión de ideas y sentimientos, y valorar la lengua oral y escrita como un medio de relación con los demás y regulación de la convivencia.

La competencia relacionada con el aprendizaje de lenguas y de lectoescritura está valorada por encima de las demás, incluyendo la utilización de recursos de animación a la lectura y la escritura, aspectos tan importantes en esta etapa educativa (sobre todo en su segundo ciclo). A nivel procedimental, favorecer las capacidades de habla y de escritura es considerado como un aspecto básico y primordial en el ámbito de la formación docente. Otra de las competencias destacada por su nivel formativo es la basada en el uso oral de una lengua extranjera para comunicarse en actividades dentro del aula, lo que pone de manifiesto el consenso existente en el aprendizaje de la segunda lengua desde las edades más tempranas, tal y como afirman aportaciones diversas (Muñoz, 2000; Álvarez, 2010, entre otras). Ello es algo prioritario en la educación del alumnado infantil.

Avanzamos hasta aquí en el camino de la investigación que nos ha emplazado al análisis (aunque sea parcial) de las competencias docentes del profesorado de Educación Infantil. Al llegar a esta fase de cierre, deseamos haber contribuido con ciertos fundamentos y principios que permitan, a otros investigadores, continuar con el debate y profundizar en esta temática tan importante y sobre la que es 
necesario desarrollar acciones investigadoras que permitan mejorar la formación inicial del maestro de Educación Infantil y responder, así, a lo que exige la propia naturaleza de sus funciones y compromisos éticos, sociales y técnicos.

$\mathrm{Y}$ es que, bajo nuestro punto de vista, sería interesante complementar este estudio introduciendo una serie de variables relacionadas con el género, edad, tiempo de experiencia docente... que nos permita hacer un análisis más estratificado. Del mismo modo, complementar el uso de los instrumentos de esta investigación, con otras técnicas de recogida de datos (observaciones y/o entrevistas), podría constituirse en una herramienta de autoevaluación y evaluación docente que, desde su enfoque formativo, permita mejorar no sólo el proceso de enseñanza, sino, y siendo lo más importante, la adquisición-dominio de competencias por parte de todos los docentes de Educación Infantil.

Con este estudio queremos ofrecer también a los maestros y maestras de la especialidad información para la reflexión sobre su acción (práctica educativa), para que busquen diferentes procedimientos de mejorar en el ejercicio de su profesión, lo que demanda profundizar en su faceta de práctico reflexivo y a asumir un mayor compromiso en su desarrollo profesional. No conviene olvidar que "la práctica reflexiva es una cultura que impregna la función docente como totalidad, es como una especie de valor que, desde lo transversal, está presente en la labor instructiva y educativa del profesor» (Cardona, 2013: 334).

Esperamos que todo esto ayude en la mejora de las prácticas de clase y motive a los docentes a pensar qué elementos formativos (competencias) es necesario adquirir (bien de inicio, bien en ejercicio) para promover esa mejora. En este sentido, nuestra investigación es, por lo tanto, una pequeña contribución al conocimiento de la temática que hemos abordado en él, y es un elemento más para seguir construyendo el siempre inacabado conocimiento o saber docente. Esto último ha de permitir al profesor enfrentar la creciente complejidad de su tarea en una sociedad compleja.

Finalmente, siendo conscientes de ser éste un tema de gran actualidad y sobre el que parece necesario continuar realizando numerosos estudios, sugerimos unas líneas de investigación que, desde nuestra perspectiva, aparecen como relevantes. En esta línea, profundizar en la adquisición y desarrollo de competencias en el proceso de formación del profesorado, tanto en la inicial (universitaria) como continua (práctica docente); potenciar en los planes de estudio aquellas metodologías centradas en el logro de competencias comunicativas, y, en general, promocionar toda investigación e innovación en competencias docentes representan iniciativas deseables en este ámbito. Es decir, ir acercándonos cada vez más al maestro indagador, investigador de su práctica con esa especie de sentido crítico al que, hace ya tiempo, se refería Stenhouse (1984). En este sentido, el presente trabajo incluye sólo una parte (algunos aspectos de la competencia comunicativa) de un proyecto investigador más ambicioso en el que estamos (el resto de competencias del colectivo profesional estudiado), pero que sienta las bases sobre las que fundamentarlo. 


\section{REFERENCIAS BIBLIOGRÁFICAS}

Álvarez, J. y Fernández-Cruz, M. (2009). Análisis descriptivo de la afectividad en el profesorado en formación de la Facultad de Ciencias de la Educación de la Universidad de Granada. Revista Lusofona de Edução, 13, 1-20.

Álvarez, M. V. (2010). El inglés mejor a edades tempranas. Pedagogía Magna, 5, 251-256.

ANECA (2004). Libro Blanco. Título de grado en magisterio, vols. 1 y 2. Madrid.

Anguera, M. T. (1995). Métodos de investigación en psicología. Madrid: Síntesis

Angulo, N. (2003). Normas de competencia en información. Biblioteconomía y Documentación, 1-16. Descargado el día 12 de septiembre de 2015. http:// www.ub.es/bid/11angul2.htm.

Barnett, R. (2005). Posibilidades y limitaciones de las competencias. Barcelona: Gedisa.

Beduwe, C. y Planas, J. (2003). Expansión educativa y mercado de trabajo. Madrid: Instituto Nacional de Empleo.

Berruto, G. (1979). La semántica. México: Nueva Imagen.

Bigas, M. y Correig, M. (Eds.) (2008). Didáctica de la Lengua en Educación Infantil. Madrid: Síntesis.

Bisquerra, R. (2004). Metodología de investigación educativa. Madrid: La Muralla.

Blanco, A. (Coord.) (2010). Desarrollo y evaluación de competencias en educación superior. Madrid: Narcea.

BOE n. ${ }^{\circ}$ 295, de 10-XII-2013, Ley Orgánica 8/2013, de 9 de diciembre para la mejora de la calidad educativa (LOMCE), pp. 97858-97921.

Buendía, L. (1999). Modelos de análisis de la investigación educativa. Sevilla: Alfar.

Bunk, G. P. (1994). La transmisión de las competencias en la formación y perfeccionamiento profesionales en la R.F.A. Revista CEDEFOP, 1-16.

Cabero, J. (Dir.) (2005). Formación del profesorado universitario en estrategias metodológicas para la incorporación del aprendizaje en red en el espacio de educación superior. Madrid: MEC, Programa de Estudios y Análisis.

Cardona, J. (2008). Problemática actual del profesorado en su función docente. Enseñanza E Teaching, 26, 29-56.

Cardona, J. (2013). Epistemología del saber docente. Madrid: UNED.

Castillo, S.; Rubio, M. J. y Cabrerizo, J. (2007). Programación por competencias. Madrid: Pearson.

Colás, M. P. y Buendía, L. (1994). Investigación Educativa. Alfar: Sevilla.

Coll, C. (2007). Las competencias en la educación escolar. Algo más que una moda y mucho menos que un remedio. Aula de Innovación Educativa (versión electrónica), 161. Descargada el 21 de octubre de 2015. http://www.grao.com/revistas/aula/161-lengua-y-expresion-plastica-las-competencias-en-la-educacion-escolar/las-competenciasen-la-educacion-escolar-algo-mas-que-una-moda-y-mucho-menos-que-un-remedio.

Delors, J. (1996). La educación encierra un tesoro: Informe a la UNESCO de la Comisión Internacional sobre la Educación para el siglo XXI. Madrid: Santillana-UNESCO.

García, F. E. y Murga, M. A. (2015). El profesorado de Educación Infantil ante el desarrollo sostenible. Necesidades formativas. Enseñanza \& Teaching, 33, 121-142.

Gonczi, A. (1996). Análisis de las tendencias internacionales y de los avances en educación y capacitación laboral basadas en normas de competencias. En Educación y capacitación basada en normas de competencias: una perspectiva internacional (pp. 38-40). México. 
JOSÉ CARDONA ANDÚJAR

FORMACIÓN EN COMPETENCIAS COMUNICATIVAS DE LOS MAESTROS DE EDUCACIÓN INFANTIL

González, J. y Wagenaar, R. (2003). Tuning educational structures in Europe. Final report phase one. Bilbao: Universidad de Deusto.

Hargreaves, A. (2005). Profesorado, cultura y posmodernidad. Cambian los tiempos, cambia el profesorado (3. ${ }^{a}$ edición). Madrid: Morata.

Irigoin, M. y Vargas, F. (2002). Competencia laboral: manual de conceptos, métodos y aplicaciones al sector salud. Montevideo: Cinterfor.

Levy-Leboyer, C. (1998). Gestión de las competencias. Barcelona: Gestión 2000.

Lomas, C. et al. (1993). Ciencias del lenguaje, competencia comunicativa y enseñanza de la lengua. España: Paidós Ibérica.

Marcelo, C. (2011). Evaluación del desarrollo profesional docente. Barcelona: Editorial Davini.

Marcelo, C. y Vaillant, D. (2009). Desarrollo profesional docente ¿Cómo se aprende a ensenar? Madrid: Narcea.

Martín, M. ${ }^{a}$ (2013). La competencia comunicativa en Educación Infantil. Valladolid: Universidad de Valladolid, SP.

Martínez, F. (2002). El cuestionario. Un instrumento para la investigación en las ciencias sociales. Barcelona: Laertes Psicopedagogía.

MEC (2006). Propuesta para la organización de las enseñanzas universitarias en España. Descargado el 25-03-2015. http://www.upv.es/entidades/OAI/info/U0319017.pdf.

Medina, A. et al. (2014). Las competencias docentes. Revista de Docencia Universitaria, 12, $1,239-267$.

Miguel, M. de (Dir.) (2005). Modalidades de enseñanza centradas en el desarrollo de competencias. Orientaciones para promover el cambio metodológico en el Espacio Europeo de Educación Superior. Madrid: MEC, Dirección General de Universidades. Programa de Estudios y Análisis.

Montero, A. (2010). Las competencias educativas. Tres riesgos y una oportunidad. Escuela, $3867,37$.

Mulder, M. et al. (2008). The concept of competence in the development of vocational education and training in selected Eu member status: a critical analysis. Revista del Currículum y Formación del Profesorado, 12, 3.

Muñoz, C. (2000). Segundas lenguas. Adquisición en el aula. Madrid: Ariel.

OIT (1998). Memoria del Director General. Actividades de la OIT 1998-1999. Ginebra.

Perrenoud, P. (2000). Construir competencias desde la escuela. Santiago de Chile: Dolmen.

Perrenoud, P. (2007). Diez nuevas competencias para enseñar (4. ${ }^{a}$ edición). Barcelona: Graó.

Pilleu, M. (2001). Competencia comunicativa y análisis del discurso. Estudios Filológicos, 36, 143-152.

Prado, J. (2011). Didáctica de la Lengua y Literatura para educar en el siglo XXI. Madrid: La Muralla.

Roegiers, X. (2007). Pedagogía de la integración. Competencias e integración de los conocimientos en la enseñanza. San José, Costa Rica: Coordinación Educativa y Cultural Centroamericana y AECI. Colección IDER.

Rué, J. y Martínez, M. (2005). Las titulaciones UAB en el Espacio Europeo de Educación Superior. Sistema Europeo de Transferencia de Créditos.

Sevillano, M. ${ }^{a}$ L. (2009). Competencias para uso de herramientas virtuales en la vida, trabajo y formación permanente. Madrid: Pearson-Prentice Hall. 
Sotomayor, C. y Walker, H. (2009). Formación continua de profesores: cómo desarrollar competencias para el trabajo escolar, experiencias y propuestas. Santiago de Chile: Editorial Universitaria.

Stenhouse, L. (1984). Investigación y desarrollo del currículum. Madrid: Morata.

Tejada, J. (1999a). Acerca de las competencias profesionales (I). Herramientas, 56, 20-30.

Tejada, J. (1999b). Acerca de las competencias profesionales (II). Herramientas, 57, 8-14.

Tejada, J. y Navío, A. (2005). El desarrollo y la gestión de competencias profesionales: una mirada desde la formación. Revista Iberoamericana de Educación, 37, 2.

Tobón, S. (2005). Formación basada en competencias. Pensamiento complejo, diseño curricular y didáctica. Bogotá: Ecoe.

Vaillant, D. (2007). Mejorando la formación y el desarrollo profesional docente en Latinoamérica. Pensamiento Educativo, 41, 2. Descargado el 20-septiembre 2015. www. ub.edu/obipd/PDF\%20docs/Formaci\%C3\%B3\%20Permanent/Educaci\%C3\%B3\%20 Primaria/Publicacions/Mejorando\%20la\%20formaci\%C3\%B3n\%20y\%20el\%20desarrollo\%20profesional\%20docente\%20en\%20Latinoam\%C3\%A9rica.\%20Vaillant,D.pdf.

Villa, A. y Poblete, M. (2007). Aprendizaje basado en competencias: una propuesta para la evaluación de las competencias genéricas. Bilbao: Mensajero-ICE Universidad de Deusto.

Zabala, A. (2007). Cómo aprender y enseñar competencias. Barcelona: Graó.

Zabala, A. y Arnau, L. (2008). 11 Ideas clave: como aprender y enseñar competencias (4. ${ }^{\mathrm{a}}$ reimpresión). Barcelona: Graó.

Zabalza, M. Á. (2003). Competencias didácticas del profesorado universitario. Diseño curricular en la universidad. Madrid: Narcea.

Zabalza, M. Á. (2005). Los cinco miuras de la convergencia europea. Crónica Universia. Descargado el 25-junio de 2015. http//www.universia.es/portada/actualidad.

\section{ANEXO I}

\section{Datos de identificación}

Por favor, indique a continuación los siguientes datos de identificación:

SEXO: .... Mujer .... Hombre....... AÑOS DE EXPERIENCIA DOCENTE:

CENTRO:

NIVEL EDUCATIVO QUE IMPARTE: Infantil

TIPO DE CENTRO: ... Público ... Concertado ... Privado

LOCALIDAD: 


\section{Cuestionario}

Reflexione sobre su práctica docente e indique cómo se percibe en el dominio de cada una de las competencias que se relacionan, teniendo en cuenta el siguiente baremo: 4. Bastante competente; 3. Competente: 2. Incompetente; 1. Muy incompetente.

\begin{tabular}{|c|c|c|c|c|}
\hline A. COMPETENCIAS ESPECÍFICAS COMUNES & 1 & 2 & 3 & 4 \\
\hline $\begin{array}{l}\text { 1. Conocer el desarrollo del lenguaje en la etapa de Educación Infantil } \\
\text { y diseñar estrategias didácticas orientadas al enriquecimiento de las } \\
\text { competencias comunicativas }\end{array}$ & & & & \\
\hline $\begin{array}{l}\text { 2. Conocer y promover el desarrollo cognitivo, social y de la } \\
\text { personalidad desde el nacimiento hasta los primeros años de la } \\
\text { escolarización obligatoria }\end{array}$ & & & & \\
\hline $\begin{array}{l}\text { 3. Ser capaz de utilizar la observación sistemática como principal } \\
\text { instrumento de evaluación global, formativa y continua de las } \\
\text { capacidades de los alumnos }\end{array}$ & & & & \\
\hline $\begin{array}{l}\text { 4. Guiarse por el "principio de la globalización" a la hora de } \\
\text { programar actividades y tareas educativas de } 0 \text { a } 6 \text { años }\end{array}$ & & & & \\
\hline $\begin{array}{l}\text { 5. Saber utilizar el juego como principal recurso didáctico, así como } \\
\text { diseñar actividades de aprendizaje basadas en principios lúdicos }\end{array}$ & & & & \\
\hline B. COMPETENCIAS DISCIPLINARES: COMUNICATIVA & 1 & 2 & 3 & 4 \\
\hline $\begin{array}{l}\text { 1. Conocer el desarrollo del lenguaje y diseñar estrategias didácticas } \\
\text { orientadas al enriquecimiento de las competencias comunicativas }\end{array}$ & & & & \\
\hline $\begin{array}{l}\text { 2. Conocer los fundamentos lingüísticos y didácticos del aprendizaje } \\
\text { de lenguas y evaluar su desarrollo y competencia comunicativa }\end{array}$ & & & & \\
\hline $\begin{array}{l}\text { 3. Conocer la literatura infantil y desarrollar estrategias para el } \\
\text { acercamiento de los niños al texto oral como escrito }\end{array}$ & & & & \\
\hline $\begin{array}{l}\text { 4. Dominar las lenguas oficiales de su comunidad y mostrar una } \\
\text { correcta producción y comprensión lingüística }\end{array}$ & & & & \\
\hline $\begin{array}{l}\text { 5. Promover la incorporación de los niños al aprendizaje funcional de } \\
\text { una lengua extranjera }\end{array}$ & & & & \\
\hline C. COMPETENCIAS PROFESIONALES & & & & \\
\hline $\begin{array}{l}\text { 1. Ser capaz de planificar conjuntamente actividades con todos los } \\
\text { docentes de este nivel, de forma que se utilicen agrupaciones } \\
\text { flexibles }\end{array}$ & & & & \\
\hline $\begin{array}{l}\text { 2. Conocer las estrategias metodológicas para desarrollar nociones } \\
\text { espaciales, geométricas y de desarrollo del pensamiento lógico }\end{array}$ & & & & \\
\hline $\begin{array}{l}\text { 3. Favorecer hábitos de acercamiento de los niños hacia la iniciación } \\
\text { a la lectura y la escritura }\end{array}$ & & & & \\
\hline $\begin{array}{l}\text { 4. Promover actividades de coordinación con los docentes del primer } \\
\text { ciclo de Educación Primaria, en el marco del proyecto educativo de } \\
\text { centro }\end{array}$ & & & & \\
\hline $\begin{array}{l}\text { 5. Promover el juego simbólico y de representación de roles como } \\
\text { principal medio de conocimiento de la realidad social }\end{array}$ & & & & \\
\hline
\end{tabular}

